Supporting Information

\title{
Synthesis and Pharmacological Characterization of Novel Glucagon- Like Peptide-2 (GLP-2) Analogues with Low Systemic Clearance
}

Kazimierz Wiśniewski*, Javier Sueiras-Diaz, Guangcheng Jiang, Robert Galyean,

Mark Lu, Dorain Thompson, Yung-Chih Wang, Glenn Croston,_Alexander Posch, Diane M. Hargrove, Halina Wiśniewska, Régent Laporte, John J. Dwyer, Steve Qi, Karthik Srinivasan, Jennifer Hartwig, Nicky Ferdyan, Monica Mares, John Kraus, Sudarkodi Alagarsamy, Pierre J. M. Rivière and Claudio D. Schteingart

Ferring Research Institute Inc., 4245 Sorrento Valley Boulevard, San Diego, California 92121, United States

Table of Contents

$\begin{array}{ll}\text { Additional pharmacological data } & \text { S2 }\end{array}$

Determination of helicity by CD S5

$\begin{array}{ll}\text { Purity assessment } & \text { S6 }\end{array}$

$\begin{array}{ll}\text { Physicochemical properties of analogues } & \text { 1,2, 4-86 }\end{array}$ 


\section{Additional pharmacological data}

Table S1. In Vitro $\mathrm{EC}_{50}$ Values for Analogues 1, 2 and 4-30 with Statistical Parameters

\begin{tabular}{|c|c|c|}
\hline \multirow[t]{2}{*}{ Compound } & hGLP-2R & hGLP-1R \\
\hline & $\mathrm{EC}_{50}(\mathrm{nM})\left(\mathrm{pEC}_{50} \pm \mathrm{SEM}\right)$ & $\mathrm{EC}_{50}(\mathrm{nM})\left(\mathrm{pEC}_{50} \pm \mathrm{SEM}\right)$ \\
\hline 1 & $0.07(10.1 \pm 0.04)$ & $520(6.29 \pm 0.03)$ \\
\hline 2 & $0.09(10.1 \pm 0.02)$ & $>1000^{a}(<6.00)$ \\
\hline 4 & $0.29(9.54 \pm 0.07)$ & $>1000^{a}(<6.00)$ \\
\hline 5 & $0.48(9.32 \pm 0.02)$ & $>1000^{a}(<6.00)$ \\
\hline 6 & $0.22(9.67 \pm 0.03)$ & $>1000^{a}(<6.00)$ \\
\hline 7 & $0.63(9.20 \pm 0.08)$ & $>1000^{a}(<6.00)$ \\
\hline 8 & $1.1(8.96 \pm 0.11)$ & $>1000^{a}(<6.00)$ \\
\hline 9 & $1.1(8.94 \pm 0.02)$ & $>1000^{a}(<6.00)$ \\
\hline 10 & $>30(<7.52)$ & $>1000^{a}(<6.00)$ \\
\hline 11 & $0.20(9.69 \pm 0.07)$ & $>1000^{a}(<6.00)$ \\
\hline 12 & $8.1(8.09 \pm 0.15)$ & $>1000^{a}(<6.00)$ \\
\hline 13 & $0.19(9.72 \pm 0.03)$ & $>1000^{a}(<6.00)$ \\
\hline 14 & $16^{f}(7.81 \pm 0.08)$ & $>1000^{a}(<6.00)$ \\
\hline 15 & $7.1(8.15 \pm 0.07)$ & $>1000^{a}(<6.00)$ \\
\hline 16 & $0.23(9.64 \pm 0.02)$ & $>1000^{a}(<6.00)$ \\
\hline 17 & $0.16(9.79 \pm 0.03)$ & $>1000^{a}(<6.00)$ \\
\hline 18 & $1.5(8.84 \pm 0.08)$ & $>1000^{a}(<6.00)$ \\
\hline 19 & $0.35(9.46 \pm 0.04)$ & $>1000^{a}(<6.00)$ \\
\hline 20 & $8.9(8.05 \pm 0.13)$ & $>1000^{a}(<6.00)$ \\
\hline 21 & $1.5(8.82 \pm 0.07)$ & $>1000^{a}(<6.00)$ \\
\hline 22 & $2.6(8.59 \pm 0.04)$ & $>1000^{a}(<6.00)$ \\
\hline 23 & $4.5(8.35 \pm 0.03)$ & $>1000^{a}(<6.00)$ \\
\hline 24 & $14(7.86 \pm 0.09)$ & $>1000^{a}(<6.00)$ \\
\hline 25 & $2.2(8.66 \pm 0.11)$ & $>1000^{a}(<6.00)$ \\
\hline 26 & $0.47(9.33 \pm 0.07)$ & $>1000^{a}(<6.00)$ \\
\hline 27 & $0.43(9.37 \pm 0.09)$ & $>1000^{a}(<6.00)$ \\
\hline 28 & $>30(<7.52)$ & $>1000^{a}(<6.00)$ \\
\hline 29 & $4.5(8.35 \pm 0.23)$ & $>1000^{a}(<6.00)$ \\
\hline 30 & $0.38(9.42 \pm 0.01)$ & $>1000^{a}(<6.00)$ \\
\hline
\end{tabular}

${ }^{a}$ No agonism up to the highest concentration $(1000 \mathrm{nM})$ tested 
Table S2. Pharmacological Profiles of Compounds 1, 2, 6 and 31-86 with Statistical Parameters

\begin{tabular}{|c|c|c|c|c|}
\hline \multirow[t]{2}{*}{ Compound } & hGLP-2R & \multicolumn{2}{|l|}{ hGLP-1R } & \multirow{2}{*}{$\begin{array}{l}\text { Rat iv PK } \\
\mathrm{CL} \pm \mathrm{SEM}(\mathrm{ml} / \mathrm{kg} / \mathrm{min})\end{array}$} \\
\hline & $\begin{array}{l}\mathrm{EC}_{50} \quad(\mathrm{nM})^{a} \\
\left(\mathrm{pEC}_{50} \pm \mathrm{SEM}\right)\end{array}$ & $\begin{array}{l}\mathrm{EC}_{50} \quad(\mathrm{nM}) \\
\left(\mathrm{pEC}_{50} \pm \mathrm{SEM}\right)\end{array}$ & $\begin{array}{l}\text { Efficacy } \pm \text { SEM } \\
(\%)\end{array}$ & \\
\hline 1 & $0.07(10.1 \pm 0.04)$ & $520(6.29 \pm 0.03)$ & $71 \pm 5$ & $25 \pm 1.1$ \\
\hline 2 & $0.09(10.1 \pm 0.02)$ & $>1000^{b}(<6.00)$ & & $9.9 \pm 0.55$ \\
\hline 6 & $0.22(9.67 \pm 0.03)$ & $>1000^{b} \quad(<6.00)$ & & $20 \pm 2.2$ \\
\hline 31 & $0.66(9.18 \pm 0.09)$ & $>1000^{b}(<6.00)$ & & $9.7 \pm 1.0$ \\
\hline 32 & $0.44(9.36 \pm 0.04)$ & $300(6.52 \pm 0.06)$ & $35 \pm 2$ & $\mathrm{NT}^{c}$ \\
\hline 33 & $0.20(9.70 \pm 0.06)$ & $>1000^{b}(<6.00)$ & & $0.73 \pm 0.05$ \\
\hline 34 & $0.15(9.82 \pm 0.02)$ & $180(6.73 \pm 0.03)$ & $44 \pm 2$ & $2.8 \pm 0.06$ \\
\hline 35 & $0.31(9.51 \pm 0.01)$ & $>1000^{b}(<6.00)$ & & $3.3 \pm 0.22$ \\
\hline 36 & $0.13(9.90 \pm 0.01)$ & $110(6.98 \pm 0.04)$ & $92 \pm 3$ & $5.6 \pm 0.09$ \\
\hline 37 & $0.29(9.54 \pm 0.02)$ & $10 \quad(7.99 \pm 0.06)$ & $87 \pm 6$ & $\mathrm{NT}^{c}$ \\
\hline 38 & $0.51(9.29 \pm 0.05)$ & $8.9(8.05 \pm 0.05)$ & $83 \pm 6$ & $0.69 \pm 0.05$ \\
\hline 39 & $0.15(9.82 \pm 0.02)$ & $16 \quad(7.81 \pm 0.07)$ & $94 \pm 7$ & $1.8 \pm 0.09$ \\
\hline 40 & $0.09(10.1 \pm 0.06)$ & $120(6.93 \pm 0.06)$ & $64 \pm 5$ & $1.2 \pm 0.01$ \\
\hline 41 & $0.16(9.80 \pm 0.001)$ & $8.8(8.05 \pm 0.02)$ & $89 \pm 6$ & $0.55 \pm 0.05$ \\
\hline 42 & $0.09(10.0 \pm 0.06)$ & $60 \quad(7.22 \pm 0.02)$ & $86 \pm 2$ & $0.51 \pm 0.01$ \\
\hline 43 & $0.11(9.95 \pm 0.05)$ & $100(6.99 \pm 0.04)$ & $36 \pm 3$ & $0.73 \pm 0.05$ \\
\hline 44 & $0.11(9.95 \pm 0.06)$ & $45 \quad(7.35 \pm 0.01)$ & $73 \pm 6$ & $0.32 \pm 0.01$ \\
\hline 45 & $0.18(9.75 \pm 0.01)$ & $32(7.49 \pm 0.04)$ & $68 \pm 6$ & $4.9 \pm 0.13$ \\
\hline 46 & $0.10(9.99 \pm 0.03)$ & $80 \quad(7.10 \pm 0.03)$ & $49 \pm 4$ & $1.1 \pm 0.05$ \\
\hline 47 & $0.09(10.0 \pm 0.03)$ & $83 \quad(7.08 \pm 0.05)$ & $39 \pm 4$ & $1.3 \pm 0.05$ \\
\hline 48 & $0.21(9.67 \pm 0.02)$ & $56 \quad(7.25 \pm 0.01)$ & $35 \pm 3$ & $0.71 \pm 0.06$ \\
\hline 49 & $0.10(10.0 \pm 0.04)$ & $40 \quad(7.39 \pm 0.04)$ & $80 \pm 3$ & $1.2 \pm 0.05$ \\
\hline 50 & $0.18(9.74 \pm 0.03)$ & $21 \quad(7.68 \pm 0.05)$ & $83 \pm 5$ & $\mathrm{NT}^{c}$ \\
\hline 51 & $1.9(8.73 \pm 0.05)$ & $39 \quad(7.41 \pm 0.05)$ & $108 \pm 5$ & $\mathrm{NT}^{c}$ \\
\hline 52 & $0.10(10.0 \pm 0.03)$ & $>1000^{b}(<6.00)$ & & $0.84 \pm 0.03$ \\
\hline 53 & $0.10(10.0 \pm 0.06)$ & $>1000^{b}(<6.00)$ & & $0.41 \pm 0.03$ \\
\hline 54 & $0.14(9.86 \pm 0.06)$ & $>1000^{b}(<6.00)$ & & $5.9 \pm 0.42$ \\
\hline 55 & $0.11(9.97 \pm 0.02)$ & $>1000^{b}(<6.00)$ & & $1.2 \pm 0.04$ \\
\hline 56 & $0.22(9.66 \pm 0.06)$ & $>1000^{b}(<6.00)$ & & $0.67 \pm 0.05$ \\
\hline 57 & $0.20(9.69 \pm 0.03)$ & $>1000^{b}(<6.00)$ & & $6.1 \pm 0.37$ \\
\hline 58 & $0.13(9.87 \pm 0.07)$ & $>1000^{b}(<6.00)$ & & $1.9 \pm 0.03$ \\
\hline 59 & $0.42(9.38 \pm 0.02)$ & $>1000^{b}(<6.00)$ & & $0.13 \pm 0.03$ \\
\hline
\end{tabular}




\begin{tabular}{|c|c|c|c|c|}
\hline \multirow[t]{2}{*}{ Compound } & hGLP-2R & \multicolumn{2}{|l|}{ hGLP-1R } & \multirow{2}{*}{$\begin{array}{l}\text { Rat iv PK } \\
\mathrm{CL} \pm \mathrm{SEM}(\mathrm{ml} / \mathrm{kg} / \mathrm{min})\end{array}$} \\
\hline & $\begin{array}{l}\mathrm{EC}_{50} \quad(\mathrm{nM})^{a} \\
\left(\mathrm{pEC}_{50} \pm \mathrm{SEM}\right)\end{array}$ & $\begin{array}{l}\mathrm{EC}_{50}(\mathrm{nM}) \\
\left(\mathrm{pEC}_{50} \pm \mathrm{SEM}\right)\end{array}$ & $\begin{array}{l}\text { Efficacy } \pm \text { SEM } \\
(\%)\end{array}$ & \\
\hline 60 & $0.11(9.94 \pm 0.03)$ & $>1000^{b}(<6.00)$ & & $0.44 \pm 0.03$ \\
\hline 61 & $0.19(9.71 \pm 0.02)$ & $>1000^{b}(<6.00)$ & & $0.43 \pm 0.02$ \\
\hline 62 & $0.13(9.90 \pm 0.03)$ & $>1000^{b}(<6.00)$ & & $0.28 \pm 0.03$ \\
\hline 63 & $0.15(9.83 \pm 0.01)$ & $>1000^{b}(<6.00)$ & & $0.50 \pm 0.01$ \\
\hline 64 & $0.35(9.46 \pm 0.04)$ & $>1000^{b}(<6.00)$ & & $\mathrm{NT}^{c}$ \\
\hline 65 & $0.08(10.1 \pm 0.05)$ & $>1000^{b}(<6.00)$ & & $0.37 \pm 0.02$ \\
\hline 66 & $0.09(10.1 \pm 0.06)$ & $>1000^{b}(<6.00)$ & & $0.30 \pm 0.02$ \\
\hline 67 & $0.07(10.2 \pm 0.04)$ & $90(7.05 \pm 0.03)$ & $58 \pm 4$ & $0.48 \pm 0.04$ \\
\hline 68 & $0.08(10.1 \pm 0.03)$ & $>1000^{b}(<6.00)$ & & $0.33 \pm 0.02$ \\
\hline 69 & $0.08(10.1 \pm 0.04)$ & $>1000^{b}(<6.00)$ & & $0.26 \pm 0.01$ \\
\hline 70 & $0.07(10.1 \pm 0.05)$ & $70(7.17 \pm 0.05)$ & $51 \pm 4$ & $0.52 \pm 0.04$ \\
\hline 71 & $0.04(10.4 \pm 0.04)$ & $130(6.90 \pm 0.02)$ & $41 \pm 7$ & $0.49 \pm 0.04$ \\
\hline 72 & $0.03(10.5 \pm 0.05)$ & $>1000^{b}(<6.00)$ & & $0.28 \pm 0.02$ \\
\hline 73 & $0.03(10.5 \pm 0.03)$ & $>1000^{b}(<6.00)$ & & $0.27 \pm 0.02$ \\
\hline 74 & $0.06(10.3 \pm 0.06)$ & $>1000^{b}(<6.00)$ & & $0.15 \pm 0.01$ \\
\hline 75 & $0.06(10.3 \pm 0.03)$ & $>1000^{b}(<6.00)$ & & $0.24 \pm 0.01$ \\
\hline 76 & $0.07(10.2 \pm 0.04)$ & $>1000^{b}(<6.00)$ & & $0.15 \pm 0.02$ \\
\hline 77 & $0.09(10.0 \pm 0.03)$ & $>1000^{b}(<6.00)$ & & $0.20 \pm 0.01$ \\
\hline 78 & $0.13(9.90 \pm 0.06)$ & $>1000^{b}(<6.00)$ & & $0.12 \pm 0.004$ \\
\hline 79 & $0.51(9.29 \pm 0.07)$ & $>1000^{b}(<6.00)$ & & $0.11 \pm 0.03$ \\
\hline 80 & $0.05(10.3 \pm 0.04)$ & $>1000^{b}(<6.00)$ & & $0.28 \pm 0.01$ \\
\hline 81 & $0.05(10.3 \pm 0.04)$ & $>1000^{b}(<6.00)$ & & $0.19 \pm 0.01$ \\
\hline 82 & $0.06(10.2 \pm 0.06)$ & $>1000^{b} \quad(<6.00)$ & & $0.17 \pm 0.004$ \\
\hline 83 & $0.10(10.0 \pm 0.11)$ & $>1000^{b}(<6.00)$ & & $0.10 \pm 0.01$ \\
\hline 84 & $0.04(10.4 \pm 0.03)$ & $>1000^{b}(<6.00)$ & & $0.27 \pm 0.01$ \\
\hline 85 & $0.03(10.5 \pm 0.07)$ & $>1000^{b}(<6.00)$ & & $0.23 \pm 0.01$ \\
\hline 86 & $0.03(10.6 \pm 0.06)$ & $>1000^{b}(<6.00)$ & & $0.28 \pm 0.003$ \\
\hline
\end{tabular}

${ }^{a}$ Compounds fully efficacious at the hGLP-2R. ${ }^{b}$ No agonism up to the highest concentration $(1000 \mathrm{nM})$ tested. ${ }^{c}$ Not tested. 
Table S3. Comparison of $\mathbf{7 3}$ and $\mathbf{2}$ Potency in Vitro in the rGLP-2R Assay and Effect in Vivo in the Small Intestine Growth Stimulation Assay at the Highest Dose Tested with Statistical Parameters

\begin{tabular}{lllll}
\hline Compound & $\begin{array}{l}\text { In vitro } \\
\text { rGLP-2R }\end{array}$ & $\begin{array}{l}\text { In vivo } \\
\text { Rat intestinal growth at dose of } \\
1000 \text { nmol/kg }\end{array}$ & Rat iv PK \\
\cline { 2 - 6 } & $\begin{array}{l}\mathrm{EC}_{50}(\mathrm{nM}) \\
(\mathrm{pEC} 50 \pm \mathrm{SEM}) \\
(\mathrm{nM})\end{array}$ & $\begin{array}{l}\text { Increase wet weight } \\
\text { over vehicle } \pm \mathrm{SEM} \\
(\%)\end{array}$ & $\mathrm{N}^{c}$ & $\begin{array}{l}\mathrm{CL} \pm \mathrm{SEM}(\mathrm{N}) \\
(\mathrm{ml} / \mathrm{kg} / \mathrm{min})\end{array}$ \\
\hline $\mathbf{2}$ & $0.08(10.1 \pm 0.03)$ & $39.0 \pm 0.9$ & 18 & $9.9 \pm 0.55(15)$ \\
$\mathbf{7 3}$ & $0.04(10.2 \pm 0.04)$ & $61.2 \pm 0.9$ & 17 & $0.27 \pm 0.02(3)$
\end{tabular}

\footnotetext{
${ }^{a}$ In vitro potency at the rat GLP-2 receptor. ${ }^{b}$ Compounds were administered once daily for 5 days, see Fig. $3{ }^{c} \mathrm{~N}$ - number of rats per dose.
}

\section{Determination of helicity by CD}

Circular dichroism spectra were collected on an Aviv Model 420 spectrophotometer. Peptides were tested in PBS 1X buffer (Mediatech, catalogue number 21-040-CM) at concentrations ranging from 5-135 $\mu \mathrm{M}$, depending on solubility. Spectra were collected at $25^{\circ} \mathrm{C}$ in a $0.1 \mathrm{~mm}$ quartz cuvette using a wavelength step of $0.5 \mathrm{~nm}$ and an averaging time of 8 seconds. The spectra for PBS was subtracted from each curve, and the data was then converted to mean residue ellipticity using the equation below, where $\mathrm{n}$ is the number of amino acids in the peptide, $\mathrm{C}$ is the molar concentration, and 1 is the path length of the cuvette in $\mathrm{cm}$. The percent helicity was calculated using software (CD Spectra Deconvolution, Version 2.1) provided with the instrument. This software takes the observed MRE spectra and deconvolutes it using basis spectra of model peptides with known secondary structure.

$$
\mathrm{MRE}=\frac{\theta_{\mathrm{obs}}(m \mathrm{deg})}{10 \times n \times C \times l}
$$


Table S4. Rat Plasma Protein Binding and Helicity Values with Statistical Parameters for Selected Compounds

\begin{tabular}{|c|c|c|c|c|c|}
\hline Compound & Major Modifications $^{a}$ & $\begin{array}{l}\text { PPB } \\
(\%)\end{array}$ & $\begin{array}{l}\text { PPB STD } \\
(\%)\end{array}$ & $\begin{array}{l}\text { Helicity } \\
(\%)\end{array}$ & $\begin{array}{l}\text { Helicity } \\
\text { STD (\%) }\end{array}$ \\
\hline 1 & None & 66.2 & 13.1 & 21 & 1.4 \\
\hline 2 & & 58.1 & 7.6 & 22 & 2.1 \\
\hline 39 & $\mathrm{Phe}^{11}, \mathrm{C}$-term $\mathrm{NH}_{2}$ & N.D. ${ }^{b}$ & N.D. ${ }^{b}$ & 47 & 2.8 \\
\hline 40 & D-Phe ${ }^{11}$, C-term $\mathrm{NH}_{2}$ & 96.1 & 0.4 & 29 & 1.4 \\
\hline 52 & $\mathrm{Leu}^{16}, \mathrm{C}$-term $\mathrm{NH}_{2}$ & 98.7 & 0.7 & 39 & 15 \\
\hline 65 & D-Phe ${ }^{11}, \mathrm{Leu}^{16}, \mathrm{C}$-term $\mathrm{NH}_{2}$ & 97.6 & 1.8 & 42 & 5.7 \\
\hline 72 & D-Phe ${ }^{11}$, Leu $^{16}$ & 99.5 & 0.1 & 29 & 5.0 \\
\hline 73 & D-Phe ${ }^{11}, \mathrm{Leu}^{16}, \mathrm{C}$-term $\mathrm{NH}_{2}$ & 99.2 & 0.3 & 27 & 5.0 \\
\hline 81 & D-Phe ${ }^{11}$, Leu $^{16}$, C-term NHEt & N.D. ${ }^{b}$ & N.D. ${ }^{b}$ & 55 & 5.7 \\
\hline 82 & D-Phe ${ }^{11}$, Leu $^{16}$, C-term NHiBu & 98.4 & 0.7 & 73 & 11 \\
\hline 83 & D-Phe ${ }^{11}$, Leu $^{16}$, C-term NHBzl & N.D. ${ }^{b}$ & N.D. ${ }^{b}$ & 75 & 7.6 \\
\hline 84 & D-Phe ${ }^{11}$, Leu $^{16}$, C-term NH-4Pic & N.D. ${ }^{b}$ & N.D. ${ }^{b}$ & 49 & 14 \\
\hline
\end{tabular}

${ }^{a}$ All compounds are the $\mathrm{Gly}^{2}$ analogues except for hGLP-2, 1. 1 and 2 are the Met ${ }^{10}$ analogues and all other compounds are the Nle ${ }^{10}$ analogues. 1, 2, 72, 73, 81-84 are 33mer peptides and 39, 40, 52 and 65 are 30 -mer peptides. ${ }^{b}$ N.D. - not determined.

\section{Assessment of final purity of analogues}

Final purity of analogues was assessed on a 1100 Agilent Liquid Chromatograph using the following analytical method: column - Vydac C18, $5 \mu \mathrm{m}, 2.1$ x $250 \mathrm{~mm}$; column temperature $-40^{\circ} \mathrm{C}$; flow rate $-0.3 \mathrm{~mL} / \mathrm{min}$; solvent $\mathrm{A}-0.01 \%$ aqueous TFA; solvent $\mathrm{B}$ $-70 \% \mathrm{CH}_{3} \mathrm{CN}, 0.01 \%$ TFA; gradient $-0-20 \% \mathrm{~B}$ in $1 \mathrm{~min}$., then $20-40 \% \mathrm{~B}$ in $20 \mathrm{~min}$., then held at $100 \%$ B for 5 min.; when necessary the first two segments of the gradient were adjusted for compound lipophilicity; UV detection at $214 \mathrm{~nm}$. The purity of most analogues exceeded $95 \%$. The purity of compounds $4,6,30,77,78,80,81$ was in the range of $92-95 \%$ and compounds $\mathbf{2 4}$ and $\mathbf{7 9}$ were $89.6 \%$ and $80.1 \%$ pure, respectively. 


\section{Determination of HPLC capacity factors}

HPLC capacity factors $\left(k^{\prime}\right)$ were calculated using the following equation: $k^{\prime}=\left(t_{R}-\right.$ $\left.t_{0}\right) / t_{0}$ where $t_{R}$ is retention time of a test compound and $t_{0}$ is retention time of unretained species. Retention times $t_{0}$ and $t_{R}$ were determined on a 1200 Agilent Liquid Chromatograph using the following analytical method: column - Phenomenex Gemini $\mathrm{C} 18,3 \mu \mathrm{m}, 2.0 \times 150 \mathrm{~mm}$; column temperature $-40^{\circ} \mathrm{C}$; flow rate $-0.3 \mathrm{~mL} / \mathrm{min}$; solvent $\mathrm{A}-0.01 \%$ aqueous TFA; solvent $\mathrm{B}-70 \% \mathrm{CH}_{3} \mathrm{CN}, 0.01 \%$ TFA; gradient $-1 \% \mathrm{~B}$ in 0.5 min., then $1-50 \%$ B in 0.5 min., then $50-65 \%$ B in 20 min., then held at $100 \%$ B for 3 min.; UV detection at $214 \mathrm{~nm}$; injection volume $-5 \mu \mathrm{L}$.

Table S5. Physicochemical Properties of Compounds 1, 2 and 4-86

\begin{tabular}{lllll}
\hline Compound & $\begin{array}{l}\text { HPLC capacity } \\
\text { factor (k') }\end{array}$ & HPLC Purity & $\begin{array}{l}\text { MH+ } \\
\text { calculated }\end{array}$ & $\begin{array}{l}\text { MH+ } \\
\text { observed }\end{array}$ \\
\hline $\mathbf{1}$ & 4.03 & 95.9 & 3764.8 & 3763.9 \\
$\mathbf{2}$ & 4.03 & 97.7 & 3750.8 & 3750.1 \\
$\mathbf{4}$ & 4.00 & 94.2 & 3634.8 & 3634.6 \\
$\mathbf{5}$ & 3.40 & 99.3 & 3533.8 & 3534.2 \\
$\mathbf{6}$ & 3.72 & 93.7 & 3420.7 & 3420.6 \\
$\mathbf{7}$ & 4.86 & 97.4 & 3292.6 & 3292.0 \\
$\mathbf{8}$ & 4.83 & 95.7 & 3191.5 & 3191.4 \\
$\mathbf{9}$ & 4.98 & 96.6 & 3063.5 & 3063.2 \\
$\mathbf{1 0}$ & 3.68 & 96 & 2837.3 & 2837.4 \\
$\mathbf{1 1}$ & 4.04 & 98.4 & 3416.7 & 3417.0 \\
$\mathbf{1 2}$ & 4.01 & 99.1 & 3402.7 & 3402.8 \\
$\mathbf{1 3}$ & 3.90 & 98.2 & 3402.7 & 3403.0 \\
$\mathbf{1 4}$ & 3.95 & 97.7 & 3402.7 & 3402.8 \\
$\mathbf{1 5}$ & 3.81 & 96.7 & 3402.7 & 3403.0 \\
$\mathbf{1 6}$ & 3.70 & 99.9 & 3402.7 & 3402.8 \\
$\mathbf{1 7}$ & 3.87 & 99.3 & 3402.7 & 3402.8 \\
$\mathbf{1 8}$ & 3.65 & 99.4 & 3402.7 & 3402.8 \\
$\mathbf{1 9}$ & 3.74 & 98.1 & 3402.7 & 3402.8 \\
$\mathbf{2 0}$ & 3.77 & 97.6 & 3402.7 & 3403.2 \\
$\mathbf{2 1}$ & 3.94 & 95.6 & 3402.7 & 3402.6 \\
$\mathbf{2 2}$ & 3.94 & 98.5 & 3402.7 & 3402.8 \\
$\mathbf{2 3}$ & 4.47 & 98.8 & 3402.7 & 3402.8 \\
$\mathbf{2 4}$ & 4.01 & 89.6 & 3402.7 & 3402.8 \\
& & & &
\end{tabular}




\begin{tabular}{|c|c|c|c|c|}
\hline Compound & $\begin{array}{l}\text { HPLC capacity } \\
\text { factor }\left(\mathrm{k}^{\prime}\right)\end{array}$ & HPLC Purity & $\begin{array}{l}\mathrm{MH}+ \\
\text { calculated }\end{array}$ & $\begin{array}{l}\mathrm{MH}+ \\
\text { observed }\end{array}$ \\
\hline 25 & 3.36 & 97.6 & 3402.7 & 3402.8 \\
\hline 26 & 4.09 & 97.9 & 3402.7 & 3402.8 \\
\hline 27 & 3.93 & 99.4 & 3402.7 & 3402.8 \\
\hline 28 & 3.83 & 97.6 & 3402.7 & 3403.0 \\
\hline 29 & 4.33 & 95.7 & 3402.7 & 3402.6 \\
\hline 30 & 4.16 & 93.8 & 3402.7 & 3402.6 \\
\hline 31 & 3.61 & 98.4 & 3388.7 & 3388.8 \\
\hline 32 & 3.68 & 98.3 & 3425.7 & 3426.0 \\
\hline 33 & 3.63 & 97.8 & 3425.7 & 3426.0 \\
\hline 34 & 4.77 & $>99.9$ & 3401.7 & 3401.8 \\
\hline 35 & 4.56 & $>99.9$ & 3401.7 & 3401.8 \\
\hline 36 & 4.71 & 99.7 & 3401.8 & 3402.0 \\
\hline 37 & 5.05 & $>99.9$ & 3415.8 & 3416.0 \\
\hline 38 & 5.50 & 99.7 & 3441.8 & 3442.0 \\
\hline 39 & 4.77 & $>99.9$ & 3435.7 & 3435.6 \\
\hline 40 & 4.86 & $>99.9$ & 3435.7 & 3435.8 \\
\hline 41 & 5.22 & 99.7 & 3469.7 & 3469.8 \\
\hline 42 & 5.14 & 98.4 & 3469.7 & 3469.8 \\
\hline 43 & 5.11 & 99.8 & 3469.7 & 3470.2 \\
\hline 44 & 5.41 & 96 & 3469.7 & 3470.2 \\
\hline 45 & 4.29 & 99.1 & 3451.7 & 3452.2 \\
\hline 46 & 4.80 & 99.1 & 3441.7 & 3442.0 \\
\hline 47 & 4.71 & 98.6 & 3441.7 & 3442.2 \\
\hline 48 & 4.72 & 98.7 & 3474.7 & 3474.8 \\
\hline 49 & 4.66 & 99 & 3474.7 & 3474.8 \\
\hline 50 & 5.68 & 99.7 & 3511.8 & 3512.0 \\
\hline 51 & 6.16 & 99.3 & 3511.8 & 3512.0 \\
\hline 52 & 4.54 & 98.8 & 3401.7 & 3401.8 \\
\hline 53 & 5.40 & 99.9 & 3441.8 & 3442.0 \\
\hline 54 & 4.55 & 98.7 & 3435.7 & 3436.2 \\
\hline 55 & 4.16 & 98.1 & 3451.7 & 3452.0 \\
\hline 56 & 4.83 & $>99.9$ & 3469.7 & 3469.8 \\
\hline 57 & 4.43 & 98.4 & 3441.7 & 3442.0 \\
\hline 58 & 3.95 & 98 & 3450.7 & 3451.0 \\
\hline 59 & 3.71 & 97.4 & 3425.7 & 3426.0 \\
\hline 60 & 4.81 & 99.8 & 3449.8 & 3449.8 \\
\hline 61 & 4.91 & 98 & 3485.8 & 3486.0 \\
\hline 62 & 5.02 & 99.6 & 3485.8 & 3486.0 \\
\hline
\end{tabular}




\begin{tabular}{|c|c|c|c|c|}
\hline Compound & $\begin{array}{l}\text { HPLC capacity } \\
\text { factor }\left(k^{\prime}\right)\end{array}$ & HPLC Purity & $\begin{array}{l}\mathrm{MH}+ \\
\text { calculated }\end{array}$ & $\begin{array}{l}\mathrm{MH}+ \\
\text { observed }\end{array}$ \\
\hline 63 & 4.53 & 98.6 & 3474.7 & 3475.0 \\
\hline 64 & 4.59 & $>99.9$ & 3511.8 & 3512.0 \\
\hline 65 & 5.48 & 98.8 & 3434.8 & 3435.2 \\
\hline 66 & 5.44 & $>99.9$ & 3468.8 & 3469.2 \\
\hline 67 & 5.12 & 99.5 & 3484.8 & 3485.2 \\
\hline 68 & 5.34 & 99.8 & 3440.7 & 3441.2 \\
\hline 69 & 5.28 & 99.3 & 3474.7 & 3475.2 \\
\hline 70 & 5.03 & 99.8 & 3490.7 & 3491.0 \\
\hline 71 & 5.48 & $>99.9$ & 3464.8 & 3465.6 \\
\hline 72 & 6.24 & 95.6 & 3764.9 & 3764.4 \\
\hline 73 & 5.98 & 96.1 & 3763.9 & 3764.2 \\
\hline 74 & 6.27 & 96.3 & 3798.9 & 3798.6 \\
\hline 75 & 5.94 & 90.2 & 3797.9 & 3797.4 \\
\hline 76 & 5.88 & 87.5 & 3803.9 & 3803.9 \\
\hline 77 & 6.96 & 92.4 & 3797.9 & 3797.3 \\
\hline 78 & 6.76 & 92.8 & 3831.9 & 3831.3 \\
\hline 79 & 7.36 & 80.1 & 3881.9 & 3881.0 \\
\hline 80 & 5.89 & 94.5 & 3799.9 & 3799.6 \\
\hline 81 & 6.35 & 94.7 & 3792.0 & 3791.8 \\
\hline 82 & 7.22 & 98.7 & 3820.0 & 3819.6 \\
\hline 83 & 7.65 & $>99.9$ & 3854.0 & 3853.6 \\
\hline 84 & 4.81 & 97.4 & 3855.0 & 3854.6 \\
\hline 85 & 4.50 & 95.1 & 4011.1 & 4010.8 \\
\hline 86 & 6.02 & 96.8 & 4082.1 & 4081.9 \\
\hline
\end{tabular}

